\title{
Prediction of species composition ratios in pooled specimens of the Anopheles Hyrcanus group using quantitative sequencing
}

Do Eun Lee ${ }^{1}$, Heung-Chul Kim², Sung-Tae Chong ${ }^{2}$,Terry A. Klein ${ }^{2}$, Ju Hyeon Kim³ ${ }^{3^{*}}$ and Si Hyeock Lee ${ }^{1,3^{*}}$ (D)

\begin{abstract}
Background: Plasmodium vivax is transmitted by members of the Anopheles Hyrcanus Group that includes six species in the Republic of Korea: Anopheles sinensis sensu stricto (s.s.), Anopheles pullus, Anopheles kleini, Anopheles belenrae, Anopheles lesteri, and Anopheles sineroides. Individual Anopheles species within the Hyrcanus Group demonstrate differences in their geographical distributions, vector competence and insecticide resistance, making it crucial for accurate species identification. Conventional species identification conducted using individual genotyping (or barcoding) based on species-specific molecular markers requires extensive time commitment and financial resources.
\end{abstract}

Results: A population-based quantitative sequencing (QS) protocol developed in this study provided a rapid estimate of species composition ratios among pooled mosquitoes as a cost-effective alternative to individual genotyping. This can be accomplished by using species- or group-specific nucleotide sequences of the mitochondrial cytochrome Coxidase subunit I (COI) and the ribosomal RNA internal transcribed spacer 2 (ITS2) region as species identification alleles in a two-step prediction protocol. Standard genomic DNA fragments of COI and ITS2 genes were amplified from each Anopheles species using group-specific universal primer sets. Following sequencing of the COI or ITS2 amplicons generated from sets of standard DNA mixtures, equations were generated via linear regression to predict species-specific nucleotide sequence frequencies at different positions. Species composition ratios between An. sineroides, An. pullus and An. lesteri were estimated from QS of the COI amplicons based on the mC.260A, mC.122C and $m C .525 \mathrm{C}$ alleles at the first step, followed by the prediction of species composition ratios between An. sinensis, An. kleini and An. belenrae based on QS of the ITS2 amplicons using the rl.370G and rl.389T alleles. The COI copy number was not significantly different between species, suggesting the reliability of CO/-based prediction. In contrast, ITS2 showed a slightly but significantly higher copy number in An. belenrae, requiring an adjustment of its predicted composition ratio. A blind test proved that predicted species composition ratios either from pooled DNA specimens or pooled mosquito specimens were not statistically different from the actual values, demonstrating that the QS-based prediction is accurate and reliable.

Conclusions: This two-step prediction protocol will facilitate rapid estimation of the species composition ratios in field-collected Anopheles Hyrcanus Group populations and is particularly useful for studying the vector ecology of Anopheles population and epidemiology of malaria.

Keywords: Anopheles Hyrcanus Group, Species composition, COI, ITS2, Quantitative sequencing

*Correspondence: biomyst5@snu.ac.kr; shlee22@snu.ac.kr

${ }^{3}$ Research Institute of Agriculture and Life Sciences, Seoul National University, Seoul 08826, Republic of Korea

Full list of author information is available at the end of the article

\section{Background}

The Anopheles Hyrcanus Group in the Republic of Korea (ROK) includes five species (Anopheles sinensis, Anopheles pullus, Anopheles kleini, Anopheles belenrae, and 
Anopheles lesteri), which cannot be identified morphologically, and another member, Anopheles sineroides, which can be identified morphologically when specimens are not damaged during the collection process [1, 2]. Species identification is important because individual species within the Hyrcanus Group overlap geographically and demonstrate differences in their seasonal distributions, vector competence, and insecticide resistance [3-5]. Based on preliminary studies, An. kleini, and An. lesteri are primary vectors of Plasmodium vivax in the ROK, whereas An. pullus, An. belenrae, and An. sinensis are poor vectors [3-5]. As An. kleini is more commonly collected near the demilitarized zone (DMZ) where the majority of malaria cases occur, the density of this vector species is a primary covariate for identifying vivax malaria risk [6]. Since the L1014F mutation of voltagesensitive sodium channel associated with pyrethroid insecticide resistance has been found only in An. sinensis, the efficacy of vector control may depend on the relative composition of vector species [7]. Therefore, information on the abundance of Hyrcanus Group member species in areas of likely malaria transmission is crucial for understanding the population dynamics of vector populations and epidemiology of malaria for the development and implementation of an efficient vector management strategies.

Since five members of the Hyrcanus Group cannot be identified morphologically, DNA barcoding based on the internal transcribed spacer 2 (ITS2) markers have been widely used for species identification [8, 9]. However, this molecular identification is based on individual genotyping requiring extensive labour and financial resources to conduct individual specimen DNA extraction, PCR, and sequencing gene fragments for large numbers of mosquitoes. Recently, detection of Plasmodium species and insecticide resistance genes have been routinely conducted using pooled mosquito specimens, where $>30$ mosquitoes are homogenized, DNA extracted, and processed for subsequent analyses $[10,11]$. If the speciesspecific molecular loci are identified and a protocol to distinguish and quantify their frequencies is developed, the DNA or specimen pooling technique can be employed to estimate the proportion of each species within a species complex.

Species-specific loci of the mitochondrial cytochrome $C$ oxidase subunit I (COI) gene and the ITS2 rRNA gene that can distinguish each member of the Hyrcanus Group present in the ROK were first identified. Subsequently, a quantitative sequencing (QS) protocol was developed to estimate the proportion of each species in pooled samples using individual species-specific nucleotide signals at multiple loci of the COI or ITS2 genes. This two-step method was shown to provide a rapid and reliable estimation of the species composition ratios for members of the Hyrcanus Group, and thus is useful for studying vector ecology and epidemiology of vivax malaria in the ROK.

\section{Methods \\ Anopheles genomic DNA extraction and target gene amplification}

Six members of the Hyrcanus Group were collected from Paju, Gyeonggi province, ROK. For molecular identification, genomic DNA (gDNA) was individually extracted using DNeasy Blood \& Tissue Kit (QIAGEN, Germany). Then, the ITS2 region of each specimen was amplified using rDNA $5.8 \mathrm{~S}$ forward (5'-TGTGAACTG CAGGACACATGAA- $\left.3^{\prime}\right)$ and rDNA $28 \mathrm{~S}$ reverse $\left(5^{\prime}\right.$ ATGCTTAAATTTAGGGGGTAGTC-3') primers [12]. The reaction mixture $(25 \mu \mathrm{l})$ contained $10 \mathrm{ng}$ of template DNA, $2 \mu \mathrm{l}$ of $2.5 \mathrm{mM}$ dNTP, $2.5 \mu \mathrm{l}$ of $10 \mathrm{X}$ buffer, $0.4 \mu \mathrm{M}$ of each primer, $0.12 \mu \mathrm{l}$ of EX Taq polymerase (Takara Biotechnology, Japan) and double distilled water $\left(\mathrm{ddH}_{2} \mathrm{O}\right)$. A 3 min preincubation at $95{ }^{\circ} \mathrm{C}$ was followed by 34 cycles at $95{ }^{\circ} \mathrm{C}$ for $20 \mathrm{~s}, 55^{\circ} \mathrm{C}$ for $30 \mathrm{~s}$, and $72{ }^{\circ} \mathrm{C}$ for $1 \mathrm{~min}$, with a final extension at $72{ }^{\circ} \mathrm{C}$ for $5 \mathrm{~min}$. PCR products were purified using a Monarch Clean up kit (New England Biolabs, USA) and sequenced using an ABI3730xl sequencer at the National Instrumentation Center for Environmental Management (NICEM, Korea). Sequences from each Anopheles specimen were submitted as queries to Basic Local Alignment Search Tool (BLAST) to search similar data in GenBank. A maximum-likelihood (ML) phylogenetic tree for the ITS2 sequences (455-492 bp) for each of the six Hyrcanus Group species was created along with the reference sequences (1624-1651 bp) obtained from GenBank using MEGA-X (ver.10.0.5) (iGEM, USA).

\section{$\mathrm{COI}$ and ITS2 sequence alignment}

Although COI gene is a generally used marker for the identification of mosquito species, ITS2 was additionally used since the differences in COI sequence were insufficient to distinguish all the sibling species within Hyrcanus group. To detect any intra-species sequence polymorphism, five to seven COI sequences and three to five ITS2 sequences of each Hyrcanus Group species were downloaded from National Center for Biotechnology Information (NCBI) (Additional file 1). COI sequences and ITS2 sequences were aligned respectively using DNAstar MegAlign software (DNASTAR Inc., USA) by ClustalW methods. The ITS2 sequences obtained from collected mosquito samples were also aligned with downloaded sequences. From the alignment data, species-specific 
Table. 1 Species-specific nucleotide sequence loci of Anopheles species in COI and ITS2

\begin{tabular}{lllllllll}
\hline COI & $m C .122$ & $m C .260$ & $m C .387$ & $m C .443$ & $m C .525$ & $m C .527$ & $m C .582$ & $m C .590$ \\
\hline An. pullus & $\mathrm{C}$ & $\mathrm{T}$ & $\mathrm{C}$ & $\mathrm{A}$ & $\mathrm{T}$ & $\mathrm{A}$ & $\mathrm{C}$ & $\mathrm{T}$ \\
An. lesteri & $\mathrm{T}$ & $\mathrm{T}$ & $\mathrm{T}$ & $\mathrm{A}$ & $\mathrm{T}$ & $\mathrm{A}$ & $\mathrm{T}$ & $\mathrm{T}$ \\
An. sineroides & $\mathrm{C}$ & $\mathrm{A}$ & $\mathrm{T}$ & $\mathrm{T}$ & $\mathrm{T}$ & $\mathrm{A}$ & $\mathrm{T}$ & $\mathrm{T}$ \\
skb & $\mathrm{T}$ & $\mathrm{T}$ & $\mathrm{T}$ & $\mathrm{A}$ & $\mathrm{C}$ & $\mathrm{T}$ & $\mathrm{T}$ \\
\hline ITS2 & $\mathrm{rl.370}$ & $\mathrm{rl.372}$ & $\boldsymbol{r l . 3 7 7}$ & $\boldsymbol{r l . 3 7 8}$ & $\boldsymbol{r l . 3 8 0}$ & $\boldsymbol{r l . 3 8 4}$ & $\boldsymbol{r l . 3 8 9}$ & $\boldsymbol{r l . 4 0 0}$ \\
\hline An. sinensis & $\mathrm{A}$ & $\mathrm{C}$ & $\mathrm{T}$ & $\mathrm{A}$ & $\mathrm{C}$ & $\mathrm{T}$ & $\mathrm{A}$ & $\mathrm{G}$ \\
An. kleini & $\mathrm{G}$ & $\mathrm{T}$ & $\mathrm{C}$ & $\mathrm{A}$ & $\mathrm{T}$ & $\mathrm{G}$ & $\mathrm{A}$ & $\mathrm{G}$ \\
An. belenrae & $\mathrm{A}$ & $\mathrm{C}$ & $\mathrm{C}$ & $\mathrm{G}$ & $\mathrm{C}$ & $\mathrm{T}$ & $\mathrm{T}$ & $\mathrm{A}$
\end{tabular}

Species-specific nucleotides were screened from CO1 and ITS2 alignment for members of the Anopheles Hyrcanus Group

${ }^{a}$ skb $=$ An. sinensis, An. kleini and An. belenrae

or group-specific nucleotide sequences were identified (Table 1).

Table. 2 Designed primer sets used for predicting relative species composition ratios

\begin{tabular}{llll}
\hline Gene & Primer name & Sequence $\left(\mathbf{5}^{\prime} \mathbf{- 3}^{\prime}\right.$ ) & Size (bp) \\
\hline COI & An_COI-F & CTTTAAGTATTCTAATTCGAGCTG & 594 \\
& An_COI-R & TAAAATWGGRTCTCCTCCTCC & \\
ITS2 & Anskb_ITS2-F & CAGACAAGTAGAAAGGGCTGT & $234^{\mathrm{a}} / 235^{\mathrm{b}} / 238^{\mathrm{c}}$ \\
& Anskb_ITS2-R & ACAAATCTGGGTAGTGTTCTCT & \\
\end{tabular}

${ }^{\mathrm{a}}$ Ank $=$ An. kleini

${ }^{\mathrm{b}} \mathrm{Anb}=$ An. belenrae

${ }^{\mathrm{c}}$ Ans $=$ An. sinensis

\section{QS primer design for the amplification of $\mathrm{CO}$ and ITS2 fragments}

A set of primers (An_COI-F and An_COI-R) were designed from the conserved sequence regions across all six Anopheles species to equally amplify the target $\mathrm{COI}$ fragments among each of the mosquito species (Table 2). For ITS2 amplification, a set of primers (Anskb_ITS2-F and Anskb_ITS2-R) were designed from the conserved sequence regions of $A n$. sinensis, $A n$. kleini, and An. belenrae to block the amplification in $A n$. sineroides, An. pullus, and An. lesteri (36 45\% sequence identity for $A n s k b \_I T S 2-\mathrm{F} ; 68.2 \%$ sequence identity for Anskb_ITS2-R).

The target DNA fragments were amplified from pooled DNA samples using the $A n_{-} C O I-\mathrm{F}$ vs. $A n_{-} C O I-\mathrm{R}$ and Anskb_ITS2-F vs. Anskb_ITS2-R primer sets, respectively. The COI amplification reaction mixture contained $20 \mathrm{ng}$ of each gDNA template, $0.2 \mathrm{mM}$ of dNTP, $2.5 \mu \mathrm{l}$ of $10 \mathrm{X}$ buffer, $0.5 \mu \mathrm{M}$ of each primer, $0.12 \mu \mathrm{l}$ of EX Taq polymerase (Takara), and $\mathrm{ddH}_{2} \mathrm{O}$ up to $25 \mu \mathrm{l}$. PCR cycling conditions included preincubation for at $95^{\circ} \mathrm{C}$ for $3 \mathrm{~min}$, followed by 32 cycles at $95^{\circ} \mathrm{C}$ for $20 \mathrm{~s}, 56^{\circ} \mathrm{C}$ for
$30 \mathrm{~s}$, and $72^{\circ} \mathrm{C}$ for $1 \mathrm{~min}$, with a final extension at $72^{\circ} \mathrm{C}$ for $5 \mathrm{~min}$. To amplify the ITS 2 fragment from three species (An. sineroides, An. pullus, and An. lesteri), the reaction mixture contained $10 \mathrm{ng}$ of gDNA template, $0.2 \mathrm{mM}$ of dNTP, $2.5 \mu \mathrm{l}$ of $10 \mathrm{X}$ buffer, $0.25 \mu \mathrm{M}$ of each primer, $4 \%$ of DMSO, $0.12 \mu \mathrm{l}$ of EX Taq polymerase, and $\mathrm{dd}_{2} \mathrm{O}$ up to $25 \mu \mathrm{l}$. PCR cycling conditions included a preincubation at $95^{\circ} \mathrm{C}$ for $3 \mathrm{~min}$, followed by 34 cycles at $95{ }^{\circ} \mathrm{C}$ for $20 \mathrm{~s}$, $64^{\circ} \mathrm{C}$ for $25 \mathrm{~s}, 72{ }^{\circ} \mathrm{C}$ for $50 \mathrm{~s}$, with a final extension at $72{ }^{\circ} \mathrm{C}$ for $5 \mathrm{~min}$.

\section{Establishment of a two-step QS protocol for estimating species composition ratios}

A two-step QS workflow was developed using PCRamplified fragments of the COI and ITS2 genes (Fig. 1). The species composition ratios between An. sineroides, An. pullus, and An. lesteri were first estimated using QS of the COI amplicons that included target DNA fragments from all the six Anopheles species, if present. The species composition ratios of An. sinensis, An. kleini, and An. belenrae were predicted using QS of the ITS2 amplicons that did not contain amplified target DNA fragments from An. sineroides, An. pullus, or An. lesteri.

Based on the characteristics of Sanger sequencing that the nucleotide signal intensity is affected by the surrounded nucleotide bases [13], equally diluted PCR products of each species were mixed in various ratios to prepare standard DNA templates for QS (Additional file 2) and sequenced to establish the nucleotide signal prediction equations based on linear regression analysis. In preparing the standard DNA templates, the proportion of An. sineroides was limited to $10-50 \%$ since collected numbers are usually $<5 \%$ of all Anopheles species collected in the ROK [14], whereas other species were mixed at $10-90 \%$ proportions. Nucleotide base signal ratios in sequencing data were analysed using Chromas (ver. 2.6.6) and linear regression analysis was done using 


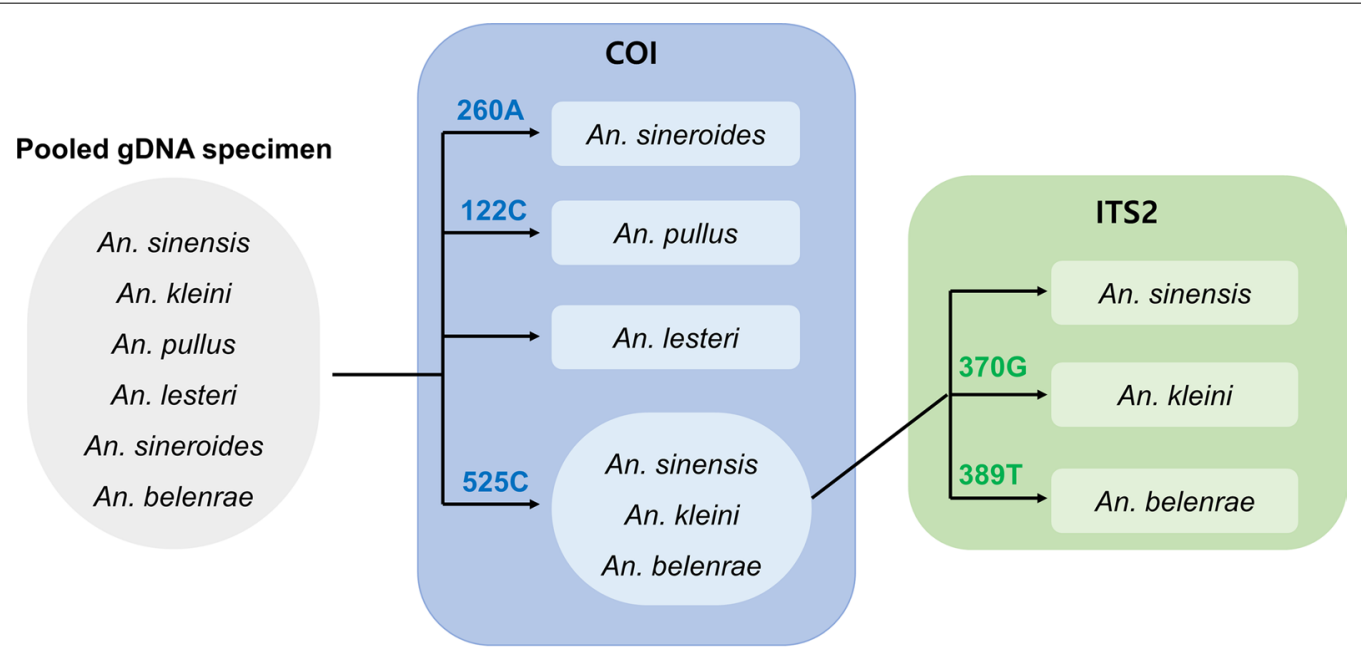

First-step using COI marker

Second-step using ITS2 marker

Fig. 1 Schematic diagram of the workflow for predicting species composition ratio. The COI fragment were amplified and sequenced from pooled gDNA specimens. Based on the sequencing chromatograms, the nucleotide signals of three COl loci were quantified to estimate the composition ratios of Anopheles sineroides, Anopheles pullus, and Anopheles lesteri. Next, the ITS2 gene fragments were selectively amplified and sequenced for Anopheles sinensis, Anopheles kleini, and Anopheles belenrae. The nucleotide signals of two ITS2 loci were quantified from the sequence chromatograms and the composition ratios of An. sinensis, An. kleini, and An. belenrae were deduced

GraphPad Prism (ver. 6, GraphPad Inc., USA). Linear regression analysis was performed for all the species-specific nucleotide loci of $\mathrm{COI}$ and ITS2 to select the most reliable locus based on the $\mathrm{R}^{2}$ criteria and standard error of estimate $\left(S_{\text {est }}\right)$, which were the statistical measures for goodness-of-fit.

\section{Determination of copy numbers of $\mathrm{COI}$ and ITS2}

Copy numbers of $\mathrm{COI}$ (or mitochondria number) and ITS2 loci were measured by quantitative real-time PCR (qPCR) using the LightCycler 96 (Roche, Basel, Switzerland). Ribosomal protein S7 (RPS7) and ribosomal protein $L 8$ (RPL8) were used as reference genes since they are known as single-copy genes $[15,16]$. gDNA was extracted from individual mosquitoes, and species was identified based on ITS2 markers [8] as described earlier. Following species identification, $3 \sim 5$ individual gDNA samples were combined and used as templates for qPCR. The qPCR reaction mixture $(10 \mu \mathrm{l})$ contained $10 \mathrm{ng}$ of gDNA template, $0.5 \mu \mathrm{M}$ of each primer (Additional file 3) and $5 \mu$ of TB Green ${ }^{\text {TM }}$ Premix Ex Taq ${ }^{\text {TM }}$ II (Takara biotechnology, Japan). qPCR for each species was conducted with $7 \sim 20$ replicates using the amplification condition of $95^{\circ} \mathrm{C}$ denaturation step for $1 \mathrm{~min}$ followed by 35 cycles of $95^{\circ} \mathrm{C}$ for $15 \mathrm{~s}, 57^{\circ} \mathrm{C}$ for $20 \mathrm{~s}$, and $72{ }^{\circ} \mathrm{C}$ for $30 \mathrm{~s}$. Melting curve analysis was conducted to confirm the integrity of PCR product by starting the melting step at $60{ }^{\circ} \mathrm{C}$ and increasing to $95{ }^{\circ} \mathrm{C}$ with a ramping temperature of
$0.2{ }^{\circ} \mathrm{C} / \mathrm{s}$. The copy number of $\mathrm{COI}$ was estimated for all the six mosquito species. In case of ITS2, however, the copy number was only determined for An. sinensis, An. kleini and An. belenrae, as ITS2 was used for the prediction of species composition ratio in the three species.

\section{Verification assay using pooled DNA and pooled mosquito specimens}

Based on the previous study that the primary Anopheles species collected in various traps in the ROK are $A n$. sinensis, An. kleini, and An. pullus, the accuracy of the QS protocol was evaluated with serial gDNA mixtures of the three species. The gDNA ratios between two species in all the three combinations (An. pullus:An. sinensis, An. pullus:An. kleini, and An. sinensis:An. kleini) were prepared at ratios of 2:8, 3:7, 4:6, 6:4, 7:3 and 8:2, and three species combinations of 2:3:5 and 1:2:7 was amplified using $C O I$ and ITS2 primer sets. The signal intensity was obtained from two nucleotide positions of COI $[\mathrm{mC} .122$ (the nucleotide number 122 of mitochondrial COI gene; the same rule of nomenclature applies hereafter) and $m C .525]$ and a single locus (rI.370; the nucleotide number of 370 for ribosomal genes in the ITS2 region; the same rule of nomenclature applies hereafter) of ITS2. Predicted composition ratios were obtained by inversely substituting the gDNA ratios into regression equations (Tables 3, 4). The observed values of the three species were calculated by the peak of the signal intensity 
chromatogram. The Pearson correlation coefficient $(r)$ and errors between observed and predicted values were calculated using GraphPad Prism (Table 5).

To confirm that the established protocol is valid with gDNA samples prepared from pooled mosquito specimens, groups of $20 \sim 24$ individual mosquitoes collected from six different collection times and two different locations (DMZ-0619, 0712, 0828, 0911; Pyeongtaek-0706, 0815) were randomly selected and pooled. gDNA was released from one wing of each specimen using DNA releasing buffer ( $5 \%$ of DMSO, $5 \%$ of PEG $200,20 \mathrm{mM}$ of $\mathrm{NaOH}$, and $1 \mathrm{mM}$ of EDTA) for species identification. In brief, each wing was incubated in $15 \mu \mathrm{l}$ of DNA releasing buffer for $20 \mathrm{~min}$ at room temperature and then diluted with $25 \mu \mathrm{l}$ of water for PCR reaction. Following species identification based on the ITS2 marker [8], the remaining mosquito bodies were combined and homogenized together by plastic pestle in liquid nitrogen. Species composition ratios were predicted by inversely substituting the gDNA ratios into regression equations and compared to actual species composition data.

\section{Results}

\section{Phylogenetic tree of collected specimens}

Based on the COI phylogenetic tree, An. sineroides, An. pullus, and An. lesteri were clearly divided into separate clusters, whereas An. sinensis, An. kleini, and An. belenrae were clustered into a large monophyletic cluster (Additional file 4). In contrast, the ITS2 phylogenetic analysis demonstrated that all the collected specimens were clearly divided into separate clusters with corresponding GenBank references (Additional file 5).

\section{Search for species-specific loci in $\mathrm{COI}$ and ITS2}

The results of COI and ITS2 sequence alignment were organized with different color codes for each species (Additional files 6, 7). Residues that match the consensus

Table. 3 Linear regression analysis ${ }^{a}$ results of species-specific nucleotide position of $\mathrm{CO}$ gene

\begin{tabular}{|c|c|c|c|c|c|c|}
\hline Locus & Species distinction & Nucleotide & $N^{b}$ & $\mathrm{R}^{2}$ & $S_{\text {est }}{ }^{c}$ & $y=f(x)$ \\
\hline$\underline{m C .260 A}$ & An. sineroides & A & 6 & 0.995 & 1.49 & $1.039 x+1.446$ \\
\hline$m C .443 T$ & An. sineroides & T & 6 & 0.993 & 1.71 & $0.9831 x+1.910$ \\
\hline$\underline{m C .122 C}$ & An. sineroidesAn. pullus & C & 14 & 0.997 & 1.78 & $\underline{0.9942 x+2.369}$ \\
\hline mC.387C & An.pullus & C & 11 & 0.995 & 2.49 & $1.051 x+0.289$ \\
\hline$m C .582 C$ & An.pullus & C & 11 & 0.985 & 4.26 & $1.027 x+1.412$ \\
\hline$\underline{m C .525 C}$ & $s k b$ & C & 11 & 0.998 & 1.51 & $1.008 x+0.0612$ \\
\hline$m C .527 \mathrm{~T}$ & $s k b$ & T & 11 & 0.998 & 1.63 & $0.9903 x-1.457$ \\
\hline$m C .590 C$ & $s k b$ & C & 11 & 0.991 & 3.24 & $0.9821 x+2.635$ \\
\hline
\end{tabular}

${ }^{a}$ Linear regression analyses for 8 nucleotide positions were done by GraphPad Prism. The selected markers and corresponding equations for species distinction are underlined

${ }^{b}$ The number of $x$ values used for regression analysis

'Standard error of estimate

Table. 4 Linear regression analysis ${ }^{a}$ results of species-specific nucleotide position of ITS2 gene

\begin{tabular}{|c|c|c|c|c|c|c|}
\hline Locus & Species distinction & Nucleotide & $N^{b}$ & $\mathrm{R}^{2}$ & $S_{\text {est }}^{c}$ & $f(x)$ \\
\hline$r 1.370 \mathrm{G}$ & An.kleini & G & 9 & 1.000 & 0.65 & $0.998 x-0.547$ \\
\hline$r l .372 T$ & An.kleini & $\mathrm{T}$ & 9 & 0.993 & 3.29 & $1.029 x-5.815$ \\
\hline rl.380T & An.kleini & $\mathrm{T}$ & 9 & 0.968 & 6.83 & $1.012 x+7.43$ \\
\hline$r .377 \top$ & An.sinensis & $\mathrm{T}$ & 9 & 0.894 & 12.4 & $0.933 x+10.0$ \\
\hline$r / 378 G$ & An.belenrae & G & 9 & 0.996 & 2.28 & $1.003 x+0.071$ \\
\hline$\underline{\mathrm{rl} .389 \mathrm{~T}}$ & An.belenrae & $\mathrm{T}$ & 9 & 0.999 & 1.33 & $\underline{0.996 x+1.038}$ \\
\hline$r l .400 \mathrm{~A}$ & An.belenrae & A & 9 & 0.865 & 14.0 & $1.042 x-3.667$ \\
\hline
\end{tabular}

${ }^{a}$ Linear regression analyses for 8 nucleotide positions were done by GraphPad Prism. The selected markers and corresponding equations for species distinction are underlined

${ }^{b}$ The number of $x$ values used for regression analysis

' Standard error of estimate 
Table. 5 Evaluation of the accuracy of the predicted composition ratios from the pooled gDNA and pooled mosquito specimens

\begin{tabular}{|c|c|c|c|c|c|c|c|}
\hline Specimen condition & Data set & $\begin{array}{l}\text { Number of data } \\
\text { points }\end{array}$ & $r^{a}$ & $p$ & $\mathrm{R}^{2}$ & $M A E^{b}$ & Min-max error \\
\hline \multirow[t]{6}{*}{ Pooled gDNA specimen } & All & 45 & 0.984 & $<0.0001$ & 0.968 & 3.87 & $0.10-12.2$ \\
\hline & $\mathrm{COl}$ & 21 & 0.977 & $<0.0001$ & 0.955 & 4.45 & $0.10-12.2$ \\
\hline & ITS2 & 30 & 0.982 & $<0.0001$ & 0.965 & 4.02 & $0.12-12.2$ \\
\hline & An.pullus & 15 & 0.992 & $<0.0001$ & 0.984 & 3.57 & $0.10-7.34$ \\
\hline & An. kleini & 15 & 0.991 & $<0.0001$ & 0.982 & 3.28 & $0.15-10.8$ \\
\hline & An. sinensis & 15 & 0.983 & $<0.0001$ & 0.966 & 4.76 & $0.12-12.2$ \\
\hline \multirow[t]{9}{*}{ Pooled mosquito specimen } & All & 36 & 0.985 & $<0.0001$ & 0.971 & 2.90 & $0.06-13.2$ \\
\hline & $\mathrm{COI}$ & 24 & 0.987 & $<0.0001$ & 0.974 & 3.59 & $0.19-13.2$ \\
\hline & ITS2 & 18 & 0.984 & $<0.0001$ & 0.968 & 2.21 & $0.06-12.8$ \\
\hline & An.pullus & 6 & 0.985 & 0.0002 & 0.970 & 4.36 & $0.17-10.9$ \\
\hline & An. kleini & 6 & 0.985 & 0.0002 & 0.971 & 2.83 & $0.06-12.8$ \\
\hline & An. sinensis & 6 & 0.990 & $<0.0001$ & 0.980 & 3.15 & $0.16-12.1$ \\
\hline & An. belenrae & 6 & 0.997 & $<0.0001$ & 0.994 & 0.66 & $0.07-1.56$ \\
\hline & An. lesteric & 6 & N/A & N/A & N/A & 3.85 & $1.51-8.13$ \\
\hline & An. sineroides & 6 & 0.984 & 0.0002 & 0.968 & 1.09 & $0.19-1.45$ \\
\hline
\end{tabular}

${ }^{a}$ Pearson correlation coefficient

${ }^{\mathrm{b}}$ Mean absolute error

' Statistical analysis of An. lesteri was not possible since all the actual value was zero

sequences were marked as dots, and black background was applied for the sequences that differ from the consensus. The locations of species-specific $C O I$ sequence loci used for species discrimination are listed in Table 1. Nucleotide sequences at seven COI loci were found to be either species- or group-specific. The $m C .122 \mathrm{C}$ (cytosine at the nucleotide number 122 of mitochondrial COI DNA) was only observed in An. pullus, and $A n$. sineroides, whereas the remaining four species had $m C .122 \mathrm{~T}$. At the $m C .260$, and $m C .443$ loci, An. sineroides was separated from other species by having adenine and cytosine, respectively, thus allowing $m C .260 \mathrm{~A}$ (adenine at the $m C .260$ locus; the same rule of nomenclature applies hereafter) and $m C .525 \mathrm{C}$ as An. sineroides-specific alleles. The cytosine nucleotide bases at both $m C .387$ and $m C .582$ nucleotide positions were only specific to $A n$. pullus, thus these alleles were used as An. pullus-specific markers. No species-specific nucleotides to An. sinensis, An. kleini, An. belenrae, or An. lesteri were found at any $C O I$ loci examined. However, group-specific nucleotides $(m C .525 \mathrm{C}, m C .527 \mathrm{~T}$, and $m C .590 \mathrm{C})$ were identical in all three species of An. sinensis, An. kleini, and An. belenrae.

Nucleotide sequence alignment of ribosomal RNA genes from An. sinensis, An. kleini, and An. belenrae demonstrated that the longest fragment without any insertion/deletion (indel) was located in the region containing 5.8S rDNA and 28S rDNA of the ITS2 region (nucleotide number 208-446 of An. sinensis). Among a total of eight ITS2 nucleotide positions specific to individual species, the $r$ I.377T (thymine at the nucleotide number 377 of ribosomal DNA ITS2 region; the same rule of nomenclature applies hereafter) was only specific to An. sinensis, whereas other two species had rI.377 C (Table 1). The nucleotide sequences at four positions (rI.370G, rI.372T, rI.380, and rI.384) were specific to An. kleini, whereas those at three nucleotide positions (rI.378G, rI.389T, and rI.400A) were specific to An. belenrae (Table 1).

The target $C O I$ fragment was equally amplified from all six species (Fig. 2A). Due to the substantial differences in the priming sequences when using the Anskb_ITS2-F and $\mathrm{R}$ primers, however, the ITS2 fragment was only amplified from An. sinensis, An. kleini, and An. belenrae but not from An. sineroides, An. pullus, and An. lesteri (Fig. 2B).

\section{Establishment of prediction equation}

Linear regression analysis was performed for all the eight species-specific nucleotide loci of $C O I$ to select the most reliable loci based on the criteria $\left(\mathrm{R}^{2}\right.$ and $S_{\text {est }}$ ) (Table 3$)$. Between the two loci specific to An. sineroides ( $m C .260$ and $m C .443)$, the $m C .260$ locus was selected as the species-specific locus for the identification of An. sineroides because it was determined to be more reliable by showing better criteria values ( 0.995 and 1.49 for $\mathrm{R}^{2}$ and $S_{\text {est }}$, respectively). In the case of two alleles specific to $A n$. pullus ( $m$ C.387C and $m C .582 \mathrm{C})$, they were excluded as species distinction alleles, since their $S_{\text {est }}$ values (2.49 at $m C .387 \mathrm{C}$ and 4.26 at $m C .582 \mathrm{C}$ ) were much larger than 


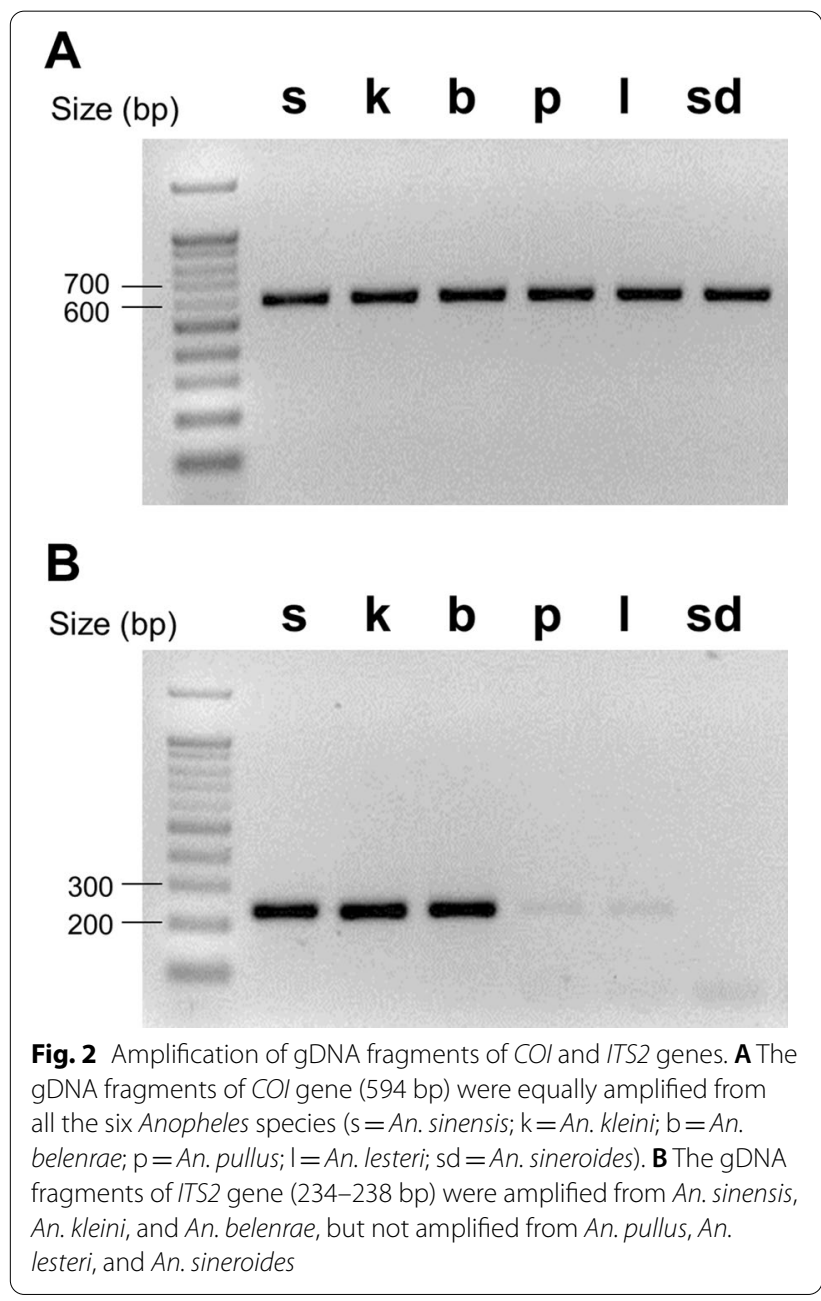

those of other loci. Instead, the $m C .122 \mathrm{C}$, showing better criteria values $\left(0.997\right.$ and 1.78 for $\mathrm{R}^{2}$ and $S_{\text {est }}$, respectively), was selected as an alleles that can simultaneously distinguish both An. pullus and An. sineroides from other species. Among the three alleles specific to the combined group of An. sinensis, An. kleini, and An. belenrae ( $m C .525 C, m C .527 \mathrm{~T}$, and $m C .590 \mathrm{C}), m C .525 \mathrm{C}$ was determined to be the best allele to estimate the combined proportion of An. sinensis, An. kleini, and An. belenrae out of six candidate species (Fig. 3A-C). Since no nucleotide position was found to be only specific to An. lesteri, the composition of An. lesteri was deduced by subtracting the combined ratios of the other five species from 1.

The same analysis and screening were performed for the seven candidate nucleotide positions (rI.384 was excluded due to the unstable signal intensity) in the ITS 2 amplicon (Table 4). Since the rI.370 nucleotide position exhibited the best criteria scores $\left(1.00\right.$ and 0.65 for $\mathrm{R}^{2}$ and $S_{\text {est }}$, respectively) for An. kleini distinction among the three nucleotide positions (rI.370, rI.372 and rI.380), the

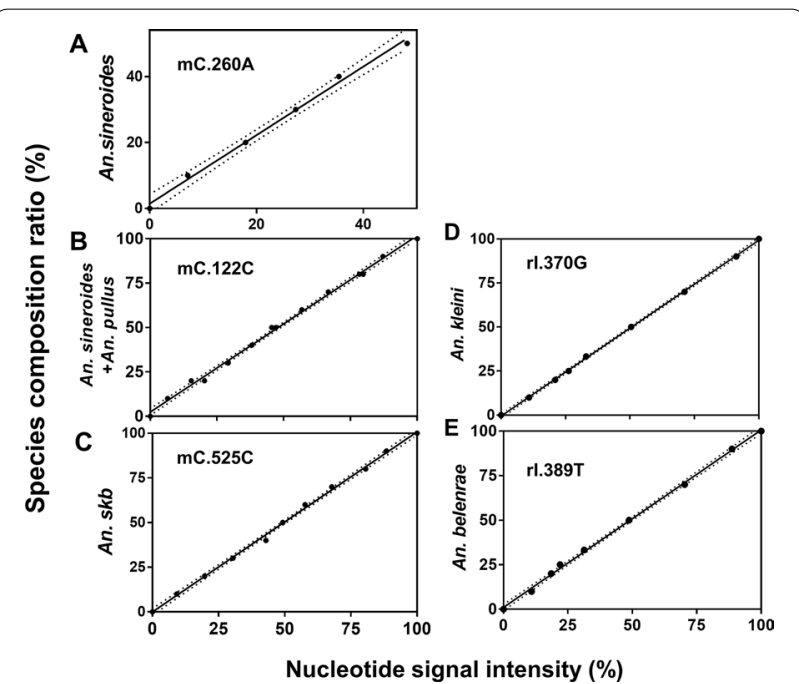

Fig. 3 Linear regression equations for the prediction of species-specific nucleotide frequencies at five different loci of $\mathrm{COI}$ and ITS2. Relationships between nucleotide signal intensities and species compositions of (A) An. sineroides, (B) An. sineroides + An. pullus, (C) An. sinensis + An. kleini + An. belenrae, (D) An. kleini, and (E) An. belenrae with $95 \% \mathrm{Cl}$ in dotted lines

rI.370G was used as the allele to estimate the ratio of $A n$. kleini. Likewise, the rI.389T allele $\left(0.999\right.$ and 1.33 for $\mathrm{R}^{2}$ and $S_{\text {est }}$, respectively) was selected out of the three alleles (rI.378G, rI.389T, and rI.400A) for estimating the proportion of An. belenrae (Fig. 3D, E). Because the An. sinensis-specific $r I .377 \mathrm{~T}$ position produced relatively lower criteria scores $\left(0.894\right.$ and 12.4 for $\mathrm{R}^{2}$ and $S_{\text {est }}$, respectively), it was not used to estimate the proportion of $A n$. sinensis. Instead, the proportion of $A n$. sinensis was calculated by subtracting the combined proportions of $A n$. kleini and $A n$. belenrae from the total proportions of $A n$. sinensis, An. kleini, and An. belenrae.

\section{Copy numbers of $\mathrm{COI}$ and ITS2 loci between species in the Anopheles Hyrcanus group}

The copy numbers of $C O I$ gene, which reflect the relative numbers of mitochondria, ranged from 69.9 ( $A n$. lesteri) to 99.8 (An. kleini), but were not significantly different between all the six Anopheles species examined $(p=0.232$; Fig. 4A). However, the average ITS2 copy number of An. sinensis, An. kleini and An. belenrae was 795, 689 and 1054, respectively. The ITS2 copy number of An. belenrae was significantly higher (1.36-fold, $p<0.0001$ ) from the other two species (Fig. 4B). Since the nucleotide signal ratio of $r I .377 \mathrm{~T}$ site can be overestimated by the copy number factor of 1.36 , the predicted value of An. belenrae composition ratio was further adjusted by multiplying the factor of 0.7 . 
A

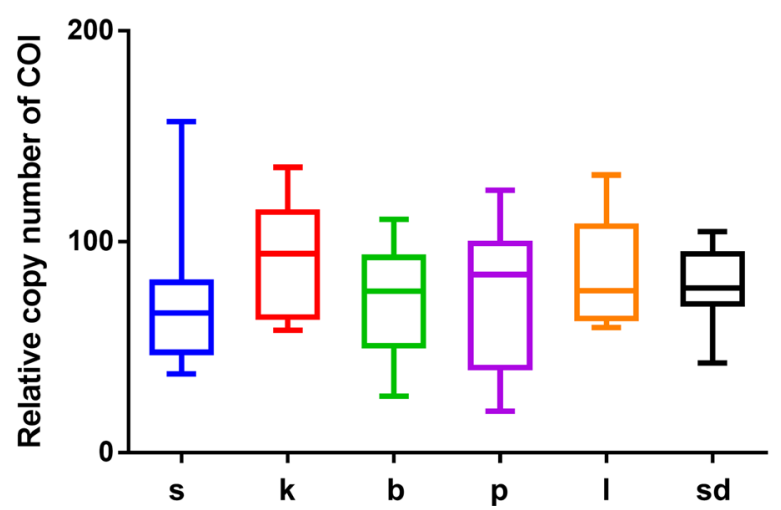

B

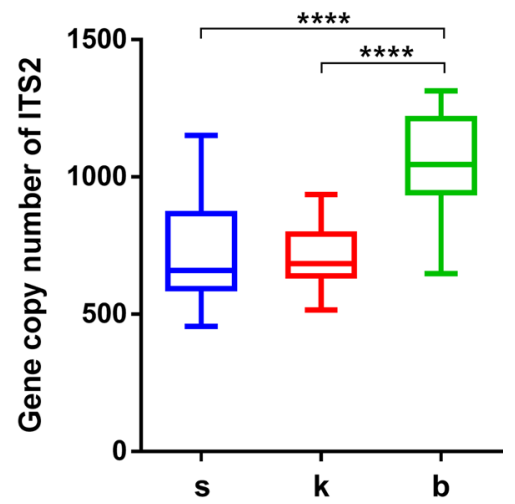

Fig. 4 Copy numbers of $\mathrm{CO}$ and ITS2 loci of Anopheles Hyrcanus Group species. A The relative copy numbers of COI locus of six Anopheles species $(s=$ An. sinensis; $\mathrm{k}=$ An. kleini; $\mathrm{b}=$ An. belenrae; $\mathrm{p}=$ An. pullus; $\mathrm{l}=$ An. lesteri; $\mathrm{sd}=$ An. sineroides). $\mathbf{B}$ The relative copy number of ITS2 locus of An. sinensis, An. kleini, and An. belenrae

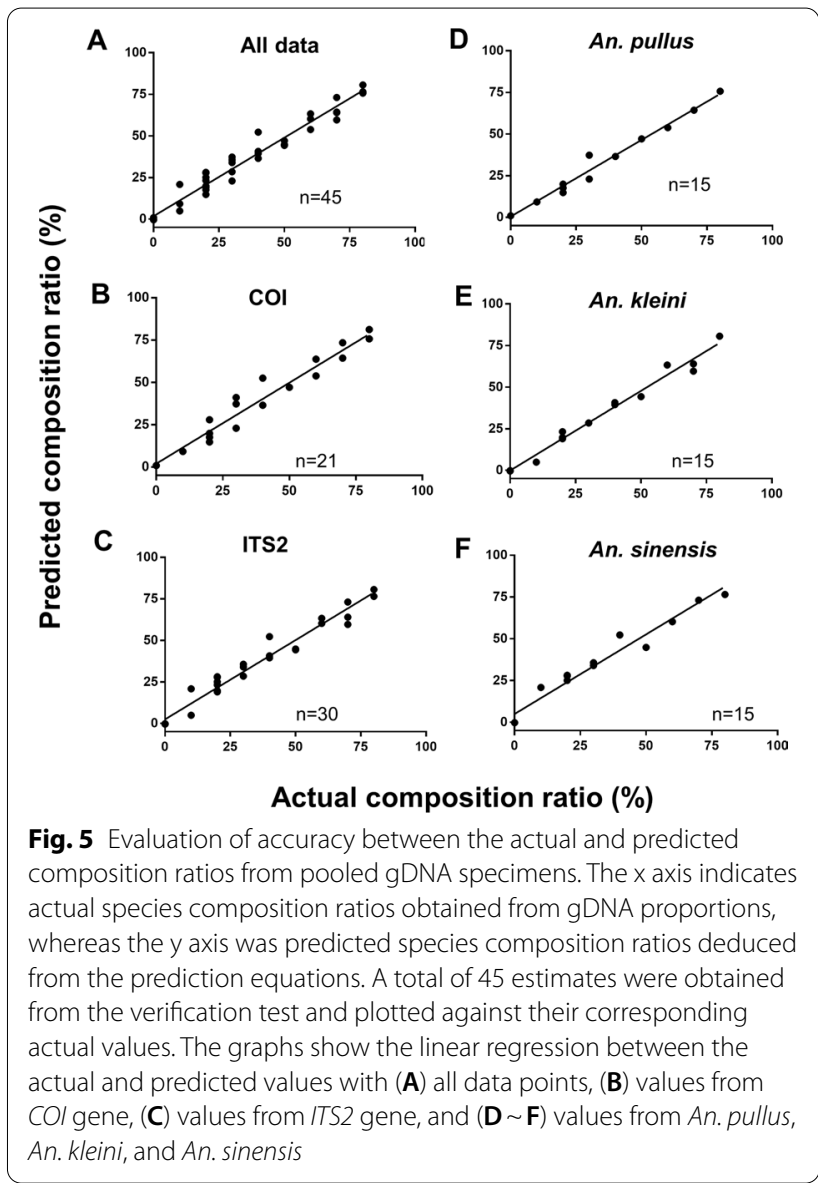

\section{Evaluation of the QS accuracy}

Validation of the QS method of three major species were performed using 15 sets of gDNA mixtures. To verify the correlation and error between the actual and predicted values, a total of 45 sets of data were plotted on a graph with an $\mathrm{x}$-axis representing the actual species composition ratio and a $y$-axis representing the estimated ratios (Fig. 5A). In addition, separate analyses were conducted for the 21 and 30 data sets derived from COI and ITS2 genes, respectively, and 15 data sets from the major species composed of three members of the Hyrcarus Group (An. pullus, An. kleini, and An. sinensis) (Fig. 5B-F). Overall data sets were confirmed to have significant correlations $\left(r>0.977, p<0.001, \mathrm{R}^{2}>0.955\right)$ between the actual and predicted composition ratios (Table 5). Mean absolute errors (MAE) of the QS prediction data of the three major species was $3.87 \%$, and MAE of COI and ITS2 loci were 4.45 and $4.02 \%$, respectively. The MAE rates for the prediction of An. pullus, An. kleini, and An. sinensis were $3.57 \%$ (maximum $7.34 \%$ ), $3.28 \%$ (maximum $10.8 \%$ ), and $4.76 \%$ (maximum $12.2 \%$ ), respectively.

Validation experiments were also conducted using gDNA samples prepared from pooled mosquito specimens. Five species (An. sinensis, An. kleini, An. pullus, An. belenrae, and An. sineroides) were found in pooled mosquito populations with composition ratios of 0:16:3:4:1, 0:9:5:6:0, 16:0:6:0:1, 1:0:9:0:0, 3:0:5:0:0 and 3:2:0:0:0, respectively, as determined by individual genotyping (Additional file 8 ). When the actual and predicted data sets were plotted on the graph (Fig. 6), the pooled mosquito data sets also showed significant correlations 

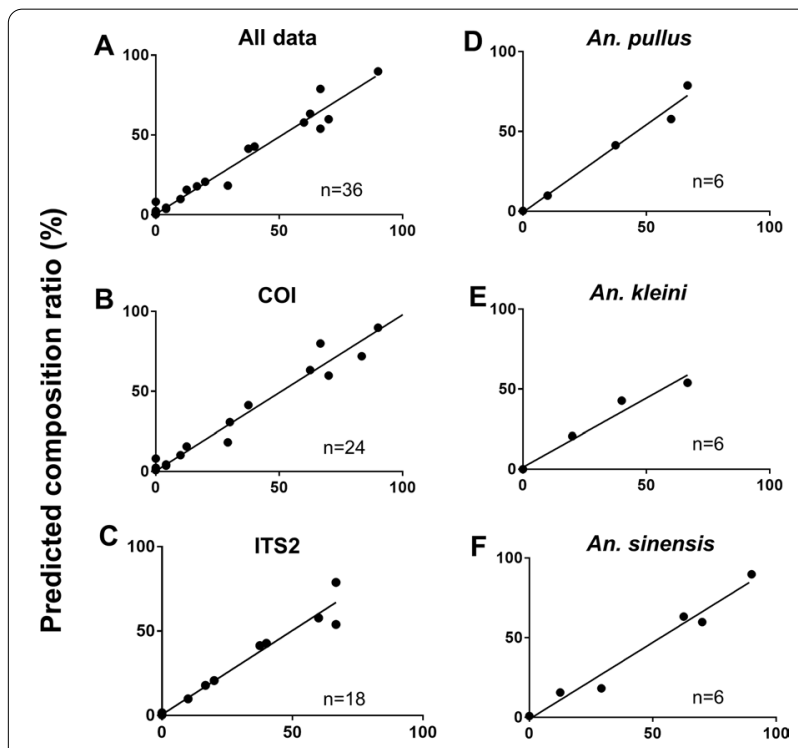

Actual composition ratio (\%)

Fig. 6 Evaluation of accuracy between the actual and predicted composition ratios from pooled mosquito specimens. The $x$ axis indicates actual species composition ratios obtained from individually identified mosquitoes in pooled mosquito specimens, whereas the $y$ axis was predicted species composition ratios deduced from the prediction equations. A total of 36 estimates were obtained from the verification test and plotted against their corresponding actual values. The graphs show the linear regression between the actual and predicted values with (A) all data points, (B) values from $\mathrm{CO}$ gene, (C) values from ITS2 gene, and (D-F) values from An. pullus, An. kleini, and An. sinensis

$\left(r>0.984, \quad p<0.0002, \quad R^{2}>0.968\right)$ between the actual and predicted composition ratios of Anopheles species (Table 5). MAE of the all data was $2.9 \%$, which is less than that of the pooled gDNA data. MAE values of $C O I$ and ITS2 were also lower (3.59 and $2.21 \%$, respectively) than those from the pooled gDNA data. Since the species with low frequencies obtains lower error rates (An. belenrae $0.66 \%$, An sineroides $1.09 \%$ ), their overall MAEs were lower in the pooled mosquito data compared to the pooled gDNA specimen data. The maximum prediction error generated from the all data set was estimated to $13.2 \%$.

\section{Discussion}

Anopheles species have been conventionally identified by morphological characteristics. However, when this method fails (e.g., for members of the Hyrcanus Group in the ROK), individual genotyping has been used to identify each species, from which the relative overall species composition was determined. As a cost-effective alternative to individual genotyping (or barcoding), a population-based QS protocol was developed that can rapidly process large numbers of mosquitoes to estimate their relative species abundance. Since similar prediction error rates were found between the pooled DNA specimens and pooled mosquito specimens, mosquito samples can be pooled and processed for QS-based prediction of species composition, as previously reported in the QSbased prediction of resistance allele frequencies in head lice [17]. Since variations in DNA content and quality between species could influence the results as main error sources, however, mosquito specimens with the same body size and quality (live or preserved specimens under identical conditions) should be used for diagnosis. In addition, copy numbers of the target loci used for species prediction should be considered as they directly determine the DNA content: if one species has more copies of the DNA loci investigated, the assay would overestimate its abundance. In this study, the mitochondrial COI alleles could be safely employed as species distinction markers as the copy number of $\mathrm{COI}$ did not differ significantly between different species in Hyrcanus group. In case of ITS2, however, An. belenrae showed slightly higher copy numbers than An. sinensis and An. kelini, requiring an additional adjustment in the prediction of its species composition. Once wild-caught Anopheles are collected, 30-100 individual mosquitoes, depending on the relative size of the collections, can be pooled (whole bodies or isolated abdomens) and processed for downstream procedures, including gDNA extraction, PCR, and sequencing. Analysis of a single pooled mosquito sample provides information on the species composition that is nearly equivalent to that obtained by the multiple numbers of individual genotyping, thereby substantially saving time and resources. For example, QS-based analysis of one pool of 100 mosquitoes requires a total cost of approximately $\$ 7$ and 1.5 days including sequencing cost and time, whereas separate individual genotyping with 100 individual mosquitoes would require much greater costs, particularly when sequencing is involved, and much longer time for gDNA extraction and downstream processes. This cost- and time-effectiveness is especially beneficial when processing large numbers of mosquitoes from different geographical locations and collection time points compared to individual genotyping.

Nevertheless, the information obtained from the QSbased prediction is not as accurate as that obtained from individual genotyping due to the prediction error. The prediction errors were on average 4.45 and $4.02 \%$ when based on COI and ITS2 genes, respectively. The respective composition ratio of An. pullus, An. lesteri, An. kleini, An. belenrae, and An. sinensis was deduced from combinations of two or three alleles $(m C .260 \mathrm{~A}$ vs. $m C .122 C, m C .122 C$ vs. $m C .525 C, m C .525 C$ vs. $r I .370 \mathrm{G}$, 
$m C .525 \mathrm{C}$ vs. $r I .389 \mathrm{~T}$ and $m C .525 \mathrm{C}$ vs. $r I .370 \mathrm{G}$ vs. rI.389T, respectively). Therefore, the error for predicting the relative ratios of these species is additive and thus becomes larger than directly predicting the composition of An. sineroides, where species composition is deduced from a single marker. Since the maximum error rates for prediction of An. pullus, An. kleini, and An. sinensis were $10.9 \%, 12.8 \%$, and $12.2 \%$, respectively, the prediction may not be accurate when the composition ratios of these species are lower than their maximum error rates. It is also worth to note that if mosquito species other than the ones investigated, such as An. koreicus, a rarely collected species, is present in a pool, the prediction results would be influenced. Considering the prediction error, this QS-based protocol is better suited as a primary survey tool to rapidly assess species composition in multiple pooled specimens in a tier system. If more accurate information on species composition for any particular mosquito sample is needed, a second round of analysis based on the conventional individual genotyping can be conducted [18].

Insecticide resistance for members of the Hyrcanus Group has been reported to be widely distributed in the ROK. Interestingly, as determined by QS-based genotyping, the resistance mutation frequencies fluctuated significantly throughout the mosquito season [11]. Frequencies of L1014F/C and G119S mutations associated with resistance to pyrethroid and organophosphorus insecticides, respectively, dramatically decreased in the Hyrcanus Group toward the fall and became zero the following spring, suggesting a possible overwintering cost associated with insecticide resistance. However, since the resistance mutation frequency was highly proportional to the composition of An. sinensis within the Hyrcanus Group ([7]; Lee DE, unpublished data), rapid estimation of the proportion of An. sinensis is crucial for understanding the resistance dynamics of Anopheles mosquitoes throughout the season. With this in mind, the high-throughput prediction of species composition based on QS using pooled DNA will facilitate the understanding of differences in insecticide resistance potential between different Anopheles spp.

The information on the geographical and seasonal distributions of Anopheles mosquitoes is crucial for establishing an efficient malaria management program. Since the species belonging to the Hyrcanus Group varies depending on geographical location and collection season, it is essential to precisely identify the relative numbers and proportion of each species over time and geographical distributions. Information on Anopheles species composition in northern Gyeonggi province in the ROK, considered as a high-risk area for vivax malaria, is particularly critical as An. kleini and An. lesteri were reported as the primary vectors with significantly high sporozoite rate and infection rate than An. sinensis $[3,19]$. Distribution and predominant species change throughout the year in northern Gyeonggi province, with $A n$. lesteri being predominant along the western coastal areas, whereas An. kleini, An. belenrae, and An. sinensis are distributed more centrally [20]. In addition, $A n$. pullus and An. belenrae are found in early summer, $A n$. kleini in mid-summer, and An. sinensis is more abundant in the late summer [14]. Therefore, a larger scale information on species composition dynamics over time and distributions would enable in-depth understanding of ecology of Hyrcanus group mosquitoes and malaria epidemiology. With this in mind, the QS protocol developed in this study should facilitate to acquire large scale phenology information, which is fundamental for assessing the impact of climate change on the malaria epidemiology in the Korean Peninsula. Moreover, the principle of QS-based prediction method can be applied to other Anopheles spp., e.g., members of the Anopheles gambiae complex of sub-Saharan Africa, to estimate the composition ratio of individual species that exhibit different seasonal occurrence, vector competence, and insecticide resistance.

\section{Conclusions}

In this study, a rapid QS-based method for the prediction of species composition ratios in pooled specimens of members of the Hyrcanus Group was developed. Since this protocol can be adapted as a cost-effective highthroughput analysis tool, rapid processing of multiple Anopheles spp. samples from multiple geographical areas and time series is feasible for large-scale studies to better understand the ecology, phenology, and epidemiology of Anopheles mosquitoes. Together with molecular tools for the detection of Plasmodium spp. and insecticide resistance, this two-step prediction protocol will facilitate to elucidate any possible correlations between vector competence and resistance potential of the Hyrcanus Group. In addition, the same principle can be applied for the quantitative analysis of species composition in other morphologically indistinguishable mosquito species complexes or groups, including the An. gambiae complex.

\section{Abbreviations}

QS: Quantitative sequencing; COl: Cytochrome Coxidase subunit l; ITS2: Internal transcribed spacer 2; ROK: Republic of Korea; DMZ: Demilitarized zone; gDNA: Genomic DNA; BLAST: Basic local alignment search tool; ML: Maximum likelihood; NCBI: National Center for Biotechnology Information. 


\section{Supplementary Information}

The online version contains supplementary material available at https://doi. org/10.1186/s12936-021-03868-y.

Additional file 1.GenBank sequences used for phylogenetic tree construction and alignments.

Additional file 2. List of amplified standard DNA mixture ratios (\%) of COI and ITS2 for regression analysis.

Additional file 3. Primer sets used for copy number determination of Anopheles Hyrcanus Group.

Additional file 4. Phylogenetic tree of $\mathrm{CO} /$ genes from NCBI sequences and collected mosquito specimens. Maximum likelihood tree with log -1673.92 score was obtained from 38 of GenBank sequences.

Additional file 5. Phylogenetic tree of ITS2 genes from NCBI sequences and collected mosquito specimens. Maximum likelihood tree with log -5366.15 score was obtained from 26 GenBank sequences and 6 collected sequences.

Additional file 6. Alignment of $\mathrm{CO}$ of Anopheles Hyrcanus Group. The partial COl gene (641 bp) sequences of six Anopheles species were aligned by ClustalW method. Red pins indicate the species-specific nucleotide sequences, and primer sites are marked as red boxes. Species arrangement was based on phylogenetic analysis of $\mathrm{CO} /$ gene in Additional file 3. Color code was given as background for each species. (s $=$ blue, An. sinensis; $\mathrm{k}=$ green, An. kleini; $\mathrm{b}=$ purple, An. belenrae; $\mathrm{l}=$ yellow, An. lesteri; $p=$ red, An. pullus; sd = gray, An. sineroides).

Additional file 7. Alignment of ITS2 of Anopheles Hyrcanus Group. The rDNA ITS2 region (450 bp) sequences of six Anopheles species were aligned by ClustalW method. Red pins indicate the species-specific nucleotide sequences, and the primer sites are marked as red boxes. Color code was the same as Additional file 5, but the order was rearranged based on the phylogenetic tree in Additional file 4.

Additional file 8 . Species composition ratios determined by individual genotyping or predicted by QS from the pooled gDNA and pooled mosquito specimens.

Acknowledgements

The authors thank to Dusim for helping collecting mosquitoes. The views expressed in this article are those of the authors and do not necessarily reflect the official policy or position of the Department of the Army, Department of Defense, or the U.S. Government. Authors, as employees of the U.S. Government (HCK, STC and TAK), conducted the work as part of their official duties. Title 17 U.S.C. $\$ 105$ provides that 'Copyright protection under this title is not available for any work of the United States Government'Title 17 U.S.C. § 101 defines a U.S. Government work is a work prepared by an employee of the U.S. Government as part of the person's official duties.

\section{Authors' contributions}

DEL did the laboratory work and wrote the manuscript. HCH, STC and TAK collected mosquito and review the manuscript. JHK and SHL coordinated the project, the study design and revised the manuscript. All authors read and approved the final manuscript.

\section{Funding}

This research was supported by the Government-wide R\&D Fund project for infectious disease research (GFID), Republic of Korea (Grant Number: HG18C0046) and the Armed Forces Health Surveillance Branch, Global Emerging Infections Surveillance and Response System (AFHSB-GEIS), Silver Spring, MD (ProMIS ID \#P0131-20-ME-03). DE Lee was supported in part by Brain Korea 21 Plus Program.

Availability of data and materials Not applicable.

\section{Declarations}

Ethics approval and consent to participate

No specific permits were required for this study. The study did not involve endangered or protected species. Therefore, the local ethics committee deemed that approval was unnecessary.

\section{Consent for publication}

All authors provided their consent for the publication of this report.

\section{Competing interests}

The authors declare that they have no competing interests.

\section{Author details}

${ }^{1}$ Department of Agricultural Biotechnology, Seoul National University, Seoul 08826, Republic of Korea. ${ }^{2}$ Medical Department Activity-Korea/65th Medical Brigade, Force Health Protection and Preventive Medicine, Unit 15281 , APO 96271-5281, AP, USA. ${ }^{3}$ Research Institute of Agriculture and Life Sciences, Seoul National University, Seoul 08826, Republic of Korea.

Received: 30 March 2021 Accepted: 27 July 2021

Published online: 06 August 2021

\section{References}

1. Rueda LM, Kim HC, Klein TA, Pecor JE, Li C, Sithiprasasna R, et al. Distribution and larval habitat characteristics of Anopheles Hyrcanus Group and related mosquito species (Diptera: Culicidae) in South Korea. J Vector Ecol. 2006:31:198-205.

2. Tanaka K, Mizusawa K, Saugstad ES. A revision of the adult and larval mosquitoes of Japan (including the Ryukyu Archipelago and the Ogasawara Islands) and Korea (Diptera: Culicidae). Army Medical Lab Pacific Apo San Francisco 96343; 1979.

3. Joshi D, Kim JY, Choochote W, Park MH, Min GS. Preliminary vivax malaria vector competence for three members of the Anopheles hyrcanus group in the Republic of Korea. J Am Mosq Control Assoc. 2011;27:312-4.

4. Ubalee R, Kim HC, Schuster AL, McCardle PW, Phasomkusolsil S, Takhampunya R, et al. Vector competence of Anopheles kleini and Anopheles sinensis (Diptera: Culicidae) from the Republic of Korea to vivax malaria-infected blood from patients from Thailand. J Med Entomol. 2016;53:1425-32.

5. Joshi D, Choochote W, Park MH, Kim JY, Kim TS, Suwonkerd W, et al. The susceptibility of Anopheles lesteri to infection with Korean strain of Plasmodium vivax. Malar J. 2009;8:42.

6. Chang KS, Yoo DH, Ju YR, Lee WG, Roh JY, Kim HC, et al. Distribution of malaria vectors and incidence of vivax malaria at Korean army installations near the demilitarized zone, Republic of Korea. Malar J. 2016;15:259.

7. Kang S, Jung J, Lee S, Hwang H, Kim W. The polymorphism and the geographical distribution of the knockdown resistance ( $k d r)$ of Anopheles sinensis in the Republic of Korea. Malar J. 2012;11:151.

8. Li C, Lee JS, Groebner JL, Kim HC, Klein TA, O'Guinn ML, et al. A newly recognized species in the Anopheles Hyrcanus Group and molecular identification of related species from the Republic of South Korea (Diptera: Culicidae). Zootaxa. 2005;939:9.

9. Fang Y, Shi WQ, Zhang Y. Molecular phylogeny of Anopheles hyrcanus group members based on ITS2 rDNA. Parasit Vectors. 2017;10:417.

10. Poolphol P, Harbach RE, Sriwichai P, Aupalee K, Sattabongkot J, Kumpitak $\mathrm{C}$, et al. Natural Plasmodium vivax infections in Anopheles mosquitoes in a malaria endemic area of northeastern Thailand. Parasitol Res. 2017:116:3349-59.

11. Lee DE, Kim HC, Chong ST, Klein TA, Choi KS, Kim YH, et al. Regional and seasonal detection of resistance mutation frequencies in field populations of Anopheles Hyrcanus Group and Culex pipiens complex in Korea. Pestic Biochem Physiol. 2020;164:33-9.

12. Cornel AJ, Porter $\mathrm{CH}$, Collins FH. Polymerase chain reaction species diagnostic assay for Anopheles quadrimaculatus cryptic species (Diptera: 
Culicidae) based on ribosomal DNA ITS2 sequences. J Med Entomol. 1996:33:109-16.

13. Carr IM, Robinson Jl, Dimitriou R, Markham AF, Morgan AW, Bonthron DT. Inferring relative proportions of DNA variants from sequencing electropherograms. Bioinformatics. 2009;25:3244-50.

14. Rueda LM, Brown TL, Kim HC, Chong ST, Klein TA, Foley DH, et al. Species composition, larval habitats, seasonal occurrence and distribution of potential malaria vectors and associated species of Anopheles (Diptera: Culicidae) from the Republic of Korea. Malar J. 2010;9:55.

15. Ruhf ML, Meister M. The Drosophila homologue of ribosomal protein L8. Insect Biochem Mol Biol. 1999;29:349-53.

16. Assogba BS, Alout H, Koffi A, Penetier C, Djogbenou LS, Makoundou P et al. Adaptive deletion in resistance gene duplications in the malaria vector Anopheles gambiae. Evol Appl. 2018;11:1245-56.

17. Kwon DH, Yoon KS, Strycharz JP, Clark JM, Lee SH. Determination of permethrin resistance allele frequency of human head louse populations by quantitative sequencing. J Med Entomol. 2008;45:912-20.
18. Clark JM. Determination, mechanism and monitoring of knockdown resistance in permethrin-resistant human head lice, Pediculus humanus capitis. J Asia Pac Entomol. 2009;12:1-7.

19. Lee WJ, Klein TA, Kim HC, Choi YM, Yoon SH, Chang KS, et al. Anopheles kleini, Anopheles pullus, and Anopheles sinensis: potential vectors of Plasmodium vivax in the Republic of Korea. J Med Entomol. 2007;44:1086-90.

20. Foley DH, Klein TA, Lee IY, Kim MS, Wilkerson RC, Harrison G, et al. Mosquito species composition and Plasmodium vivax infection rates on Baengnyeong-do (Island), Republic of Korea. Korean J Parasitol. 2011;49:313-6.

\section{Publisher's Note}

Springer Nature remains neutral with regard to jurisdictional claims in published maps and institutional affiliations.
Ready to submit your research? Choose BMC and benefit from:

- fast, convenient online submission

- thorough peer review by experienced researchers in your field

- rapid publication on acceptance

- support for research data, including large and complex data types

- gold Open Access which fosters wider collaboration and increased citations

- maximum visibility for your research: over $100 \mathrm{M}$ website views per year

At BMC, research is always in progress.

Learn more biomedcentral.com/submissions 